\title{
New visuospatial associations by training verbospatial mappings in the first language
}

\author{
Wim Notebaert, Wendy De Moor, Wim Gevers, ANd Robert J. HARTSUiker \\ Ghent University, Ghent, Belgium
}

\begin{abstract}
We investigated whether verbospatial and visuospatial information share a common representation. We demonstrate that when the associations from spatial words to spatial responses are altered, so that the word LEFT becomes associated with a right response and the word RIGHT with a left response, the associations from the spatial locations left and right to the spatial responses also change. This effect was only observed for first-language spatial words (Dutch) and not for second-language spatial words (French), and it did not increase when these languages were combined. The findings argue for shared spatial representations for different types of spatial input.
\end{abstract}

An important issue in the study of perception and action is the relationship between the representations of different types of spatial information (e.g., arrows, locations, spatial words). Spatial compatibility effects have been observed for several kinds of spatial information, suggesting similar processing pathways (Fitts \& Seeger, 1953). A spatial compatibility effect is the observation that reaction times (RTs) are faster and error percentages lower when participants make a spatially compatible response to a stimulus (left stimulus location requires left response and right stimulus location requires right response), relative to an incompatible mapping condition. Similar effects have been observed when spatial information is task-irrelevant. For instance, when participants press a left or right response key depending on the stimulus color, RTs are faster when the irrelevant stimulus location (left or right) corresponds to the response location. This is typically referred to as the Simon effect (Simon, 1990).

Whether the spatial information is activated by arrows, words, locations, or even number magnitude, the perceptual left seems to be associated with the motor left. In this study, we investigated whether different types of spatial information activate a shared spatial representation, instead of directly activating a spatially corresponding response. These shared representations introduce an intermediate or semantic level between the perceptual input and the responses.

Intermediate codes are assumed in the computational model of Zhang, Zhang, and Kornblum (1999) in order to explain (spatial) Stroop effects. When two spatial inputs activate the same representation (upward arrow presented above fixation), RTs are fast and error percentages low, as compared with when the two sources activate different representations (e.g., an upward arrow presented below fixation; see, e.g., Liu, Banich, Jacobson, \& Tanabe, 2004). In the model of Zhang et al., this effect is explained in terms of possible competition between two intermediate representations. Note that other models for Stroop effects do not implement intermediate codes (e.g., Cohen, Dunbar, \& McClelland, 1990).

Although the implementation of intermediate shared representations results in an elegant architecture, surprisingly few efforts have been made to test this hypothesis. In this study, we tested the hypothesis of intermediate spatial representations at which different types of spatial information converge. Particularly, we asked whether verbospatial (the words LEFT and RIGHT) and visuospatial information (left and right screen positions) share a common intermediate code.

We used a paradigm that was introduced by Marble and Proctor (2000) and Proctor, Marble, and Vu (2000). In this paradigm, participants switch between different tasks as a function of the stimuli. For example, Marble and Proctor instructed participants to respond spatially incompatibly to the location of white stimuli (the inducer task) and to respond left or right on the basis of the color of green and red stimuli (the diagnostic task). The green and red stimuli were also presented on the left or right of fixation, but the location was irrelevant. Hence, the colored stimuli were presented in typical Simon trials. Marble and Proctor observed a reversed Simon effect. The fact that participants responded incompatibly to the white stimuli apparently changed the association between spatial locations and spatial responses.

Using this paradigm, Notebaert, Gevers, Verguts, and Fias (2006) recently obtained evidence that the spatial information conveyed by numbers and locations converges on a shared representation. Dehaene, Bossini, and Giraux (1993) demonstrated that small numbers are associated with left responses and large numbers with right responses. Notebaert et al. showed that when participants responded magnitude incompatibly to (left on large numbers and

W. Notebaert, wim.notebaert@ugent.be 
right on small numbers), the Simon effect reversed. This clearly supports the hypothesis of shared spatial representations for numbers and space. When a new association between small numbers and right responses (instead of left responses) is created, left locations also become associated with right responses. It is important to note that Notebaert et al. only observed a reversed Simon effect in the first half of their experiment, suggesting a diminishing influence of the incompatible mapping.

Proctor et al. (2000) already mixed verbospatial and visuospatial information. In their experiment, native speakers of English responded incompatibly to the English spatial words LEFT and RIGHT (the inducer task). The diagnostic task was the standard Simon task, with red and green dots appearing on the left and right of the fixation point. Proctor et al. found neither a reversed Simon effect nor a standard Simon effect. Given the large number of replications of the Simon effect in the literature, the $a b$ sence of the Simon effect in Proctor et al.'s study suggests an influence of the inducer task, which eliminated, but did not reverse, the Simon effect. If this is so, the results can be taken as initial support for common verbospatial and visuospatial codes.

More support for shared visuospatial and verbospatial representations was provided by De Houwer, Beckers, Vandorpe, and Custers (2005). They observed an effect of a verbospatial mapping rule on visuospatial processing, but only in a condition in which Dutch (L1; native language) and French (L2; second language) words were combined in the inducer trials. De Houwer et al. therefore claimed that Proctor et al.'s (2000) failure to find a reversed Simon effect resulted from the use of only one language in the inducer trials, under the assumption that participants respond on the basis of nonsemantic (e.g., perceptual) stimulus properties when only one language is used.

In this study, we reassessed whether visuospatial and verbospatial types of information share a common representation. We used Proctor et al.'s (2000) paradigm, but adapted it in two ways. First, because Notebaert et al. (2006) showed a dissipation of the reversed Simon effect during the course of the experiment (i.e., an interaction of the Simon effect with block), we measured the Simon effect in four blocks of the experiment. Second, we implemented both single-language and mixed-language conditions in order to test whether stronger effects can be obtained in mixed-language conditions (De Houwer et al., 2005). In one language condition, participants responded incompatibly to the Dutch words LINKS ("left") and RECHTS ("right"). Because all participants had Dutch as their L1, this was labeled the L1 condition. In another language condition, we investigated the reversal of the Simon effect by practicing an incompatible mapping rule on words from an L2. We therefore used the French words GAUCHE ("left") and DROITE ("right"). In a final language condition, we combined L1 and L2, so that participants had to respond with the right response key on LINKS and GAUCHE and with the left response key on RECHTS and DROITE.

Figure 1 shows our interpretation of the computational model of Zhang et al. (1999) for spatial information. We assume that the words LINKS/GAUCHE and RECHTS/DROITE activate the corresponding semantic representations "left" and "right" ( $\mathrm{L}$ and $\mathrm{R}$ in Figure 1), even when participants respond spatially incompatibly. We expect that by using the incompatible mapping rule, the associations between the semantic representation and the spatial responses will change (the dotted lines in Figure 1). In other words, we predict that the intermediate code "left" will become associated with the right response and the intermediate code "right" will become associated with left response. When physical locations activate the same intermediate codes "right" and "left," we expect that right locations will prime the left response and left locations the right response. If this happens, a reversed Simon effect is expected when participants respond to the color of laterally presented stimuli.

The dotted lines between L 2 words and the intermediate codes indicate our assumption of reduced automatic semantic activation on the basis of L2 words. This assumption is in line with the weaker Stroop effect that has been observed for L2 words (Dyer, 1971). Although these experiments showed interference from L2 color words while naming the colors in L1, the effect is typically $75 \%$ of the size of solely L1 within-language interference (MacLeod, 1991). This is consistent with Kroll and Stewart's (1994) influential revised hierarchical model of bilingual memory. On this account, both L1 and L2 words are connected to a shared system of lexical concepts, but the connection between L2 words and concepts (and vice versa) is much weaker than the connection between L1 words and concepts.

On the basis of the presumed activation of the intermediate semantic representation, we predict that the reversed

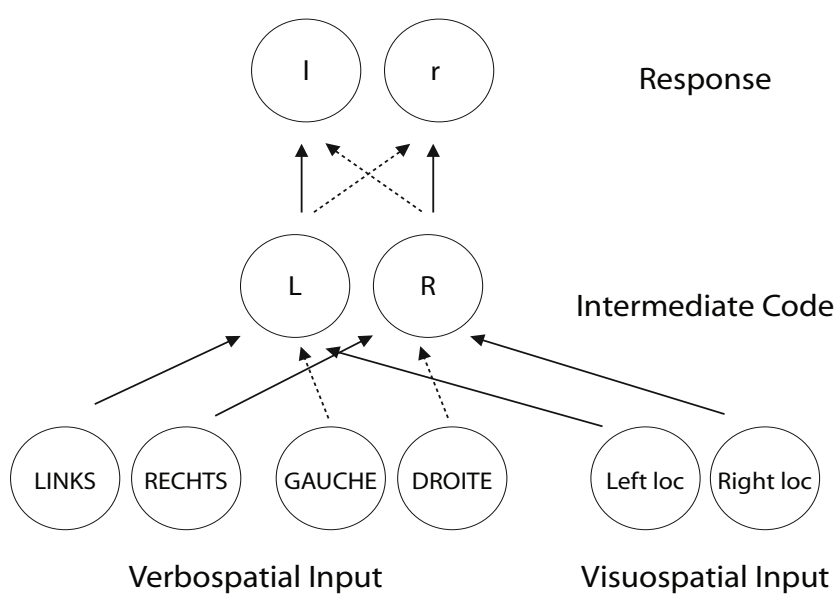

Figure 1. An architecture in which verbospatial information (the words LINKS/GAUCHE and RECHTS/DROITE) and visuospatial information activate an intermediate shared spatial representation ( $L$ or $R$ ), on the basis of the model for Stroop effects by Zhang, Zhang, and Kornblum (1999). In line with existing models for bilingualism (Kroll \& Stewart, 1994), we assume weaker semantic activation from $L 2$ words than from $L 1$ words. The intermediate representations are connected to the left (I) and right (r) responses. The solid lines represent the preexisting compatible associations, and the dotted lines represent the incompatible associations that are created through practice with the incompatible mapping rule. 
Simon effect will be less pronounced in the L2 condition than in the L1 condition. On the other hand, it is possible that also in the $\mathrm{L} 1$ condition participants might respond on the basis of perceptual features of the word stimuli, without activating semantics. This could lead to a regular Simon effect in both conditions in which only one language is used (L1 and L2). This strategy, however, is suboptimal in the combined L1/L2 condition (De Houwer et al., 2005), in which we expect a pronounced reversed Simon effect.

\section{METHOD}

\section{Participants}

Forty-five students ( 31 females and 14 males) participated in return for course credit. They were native Dutch speakers who were born in Belgium (where Dutch and French are official languages) and had been taught French from primary school onward, starting on average at the age of 10 years. Fifteen participants were randomly assigned to each of the three conditions.

\section{Apparatus and Stimuli}

Stimuli were presented on Pentium computers using E-Prime software and response boxes. The participants were seated approximately $50 \mathrm{~cm}$ from the color monitor. For all stimuli, the background was silver. Words were presented in 40-point Arial, black, bold, and uppercase, at the center of the screen. The words were approximately $6 \mathrm{~cm}$ wide and $1.2 \mathrm{~cm}$ high. The colored dots (red or green) were the letter "o" also presented in 40-point Arial bold. The stimuli were approximately $0.6 \mathrm{~cm}$ wide and $1 \mathrm{~cm}$ high and were presented $3 \mathrm{~cm}$ to the left or $3 \mathrm{~cm}$ to the right of the center of the screen.

\section{Procedure}

Depending on the language condition, the words LINKS, RECHTS, GAUCHE, and DROITE appeared centrally. Participants always had to respond spatially incompatibly. In the L1 condition, only the words LINKS and RECHTS were presented; in the L2 condition, only the words GAUCHE and DROITE were presented; and in the combined L1/ L 2 condition, the words LINKS, RECHTS, GAUCHE, and DROITE were presented. For each language condition, each word was presented an equal number of times. Irrespective of the language condition, half of the trials were words and half of the trials were colored dots (Simon trials). The mapping rule on Simon trials was counterbalanced so that for half of the participants, red was associated with the left response and green with the right response. This was reversed for the other half of the participants. The colored dots randomly appeared on the left or right of the screen. The sequence of Simon trials and word trials was completely random.

During (and only during) the response-stimulus interval, a fixation cross was presented. The interval was fixed at $550 \mathrm{msec}$. When participants made an error, the screen turned red for $100 \mathrm{msec}$, and the fixation cross was presented for $450 \mathrm{msec}$ instead of $550 \mathrm{msec}$.
Participants completed four blocks of 176 trials (88 Simon trials and 88 verbospatial incompatible trials). After each block, they had the opportunity to take a break. By pressing a response button, they started a new block of trials. No practice trials were presented, and participants were explicitly told so.

\section{RESULTS}

In the RT analyses, errors and trials following an error were excluded. One participant in the L2 condition made $39 \%$ errors on the Simon trials, and the data of this participant were not included in the analyses.

\section{Inducer Trials}

For these results, see Table 1.

Reaction times. Median RTs were analyzed with one between-subjects factor (language condition) and one within-subjects factor (block). We opted for median RTs in all analyses because such data do not require arbitrary cutoff procedures (see also Notebaert et al., 2006). For the RTs, there was a marginally significant effect of language condition $[F(2,41)=2.87, p=.07]$. Planned comparisons revealed no difference between $\mathrm{L} 1$ and $\mathrm{L} 2[F(1,41)<1$, n.s.]. There was a significant difference between $\mathrm{L} 1$ and the combined L1/L2 condition $[F(1,41)=5.56, p<.05]$ but no difference between L2 and the combined L1/L2 condition $[F(1,41)=2.33, p=.13]$.

There was also an effect of block $[F(3,123)=35.01$, $p<.001]$, indicating that RTs decreased as the experiment proceeded. Block did not interact with language condition $[F(6,123)=1.08, p=.38]$, indicating a similar decrease in RTs in the three language conditions.

Within the L1/L2 condition, we conducted an analysis to assess the performance on L1 and L2 words separately. Participants were significantly faster on L1 words than on L2 words $[F(1,14)=9.34, p<.001]$. There was also an effect of block $[F(3,42)=12.90, p<.001]$, and the interaction between language and block was significant $[F(3,42)=4.10, p<.05]$, suggesting a stronger decrease for L2 words.

Error percentages. The error percentages showed no effect of language condition $[F(2,41)=1.04, p=.36]$, but there was an effect of block $[F(3,123)=9.65, p<$ $.001]$. The interaction between the two factors was not significant $[F(6,123)<1$, n.s.]. Within the $\mathrm{L} 1 / \mathrm{L} 2$ condition, more errors were made on L2 words than on L1 words

Table 1

Reaction Times (RTs, in Milliseconds) and Error Percentages (ER\%) for the Inducer Tasks of the L1, L2, and Combined L1/L2 Conditions, Separately for the Four Blocks

\begin{tabular}{|c|c|c|c|c|c|c|c|c|c|c|c|c|}
\hline & \multicolumn{4}{|c|}{ L1 } & \multicolumn{4}{|c|}{ L2 } & \multicolumn{4}{|c|}{$\mathrm{L} 1 / \mathrm{L} 2$} \\
\hline & RT & $S E$ & ER\% & $S E$ & RT & $S E$ & ER\% & $S E$ & RT & $S E$ & ER\% & $S E$ \\
\hline Block 1 & 703 & 43 & 13 & 2 & 762 & 44 & 11 & 2 & 850 & 43 & 9 & 2 \\
\hline Block 2 & 647 & 31 & 8 & 1 & 700 & 32 & 9 & 1 & 736 & 31 & 6 & 1 \\
\hline Block 3 & 621 & 32 & 9 & 1 & 637 & 33 & 7 & 1 & 692 & 32 & 6 & 1 \\
\hline Block 4 & 590 & 28 & 6 & 1 & 597 & 24 & 7 & 1 & 679 & 28 & 5 & 1 \\
\hline \multicolumn{13}{|c|}{ Within the $\mathrm{L} 1 / \mathrm{L} 2$ Conditions } \\
\hline Block 1 & 798 & 32 & 7 & 2 & 937 & 58 & 11 & 2 & & & & \\
\hline Block 2 & 715 & 26 & 5 & 1 & 770 & 40 & 7 & 2 & & & & \\
\hline Block 3 & 669 & 25 & 4 & 1 & 721 & 34 & 7 & 1 & & & & \\
\hline Block 4 & 662 & 21 & 5 & 1 & 723 & 38 & 5 & 2 & & & & \\
\hline
\end{tabular}


$[F(1,14)=6.12, p<.05]$, and errors decreased as the experiment proceeded $[F(3,42)=3.14, p<.05]$. The interaction between block and language was not significant $[F(3,42)=1.27, p=.30]$.

\section{Diagnostic Trials}

For these results, see Table 2 and Figure 2.

Reaction times. Median RTs were analyzed using an ANOVA with one between-subjects factor (language condition) and two within-subjects factors (congruency and block). Overall, there was no RT difference for Simon trials for the three language conditions $[F(2,41)=1.14, p=$ .33]. In general, there was no Simon effect $[F(1,41)<1]$, but more importantly, as can be seen from Figure 2, the Simon effect differed across the three conditions $[F(2,41)=4.89, p<.05]$. Planned comparisons revealed a significant reversed Simon effect in the L1 condition $[F(1,41)=4.48, p<.05]$, a significant regular Simon effect in the L2 condition $[F(1,41)=4.59, p<.05]$, and no Simon effect in the combined L1/L2 condition $[F(1,41)=$ $1.13, p=.29]$.

The overall effect of block was significant $[F(3,123)=$ $37.66, p<.001]$, indicating that RTs sped up as the experiment proceeded. This was not different across the three language conditions $[F(6,123)<1$, n.s. $]$.

The Simon effect interacted with block $[F(3,123)=$ $3.68, p<.05]$. Table 2 shows that for all language conditions, RTs decreased more in congruent than in incongruent trials. The three-way interaction was not significant $[F(6,123)=1.07, p=.39]$.

Error percentages. There were no significant differences in error percentages between the three language conditions $[F(2,41)=1.45, p=.25]$. There was no overall Simon effect $[F(1,41)<1$, n.s. $]$, but the Simon effect did interact with language condition $[F(2,42)=4.79, p<$ .05]. Planned comparisons revealed no Simon effect in L1 $[F(1,41)=2.25, p=.14]$, a marginally significant regular Simon effect in L2 $[F(1,41)=3.66, p=.06]$, and a significant reversed Simon effect in L1/L2 $[F(1,41)=$ $4.83, p<.05]$.

The effect of block was significant $[F(3,123)=5.39$, $p<.01]$. Block did not interact with language condition $[F(6,123)<1$, n.s. $]$. The Simon effect in the error percentages also interacted with block $[F(3,123)=3.97, p<$
$.01]$, and the three-way interaction was not significant $[F(6,123)<1$, n.s. $]$.

Although some differences between the Simon effects in RTs and in error percentages were obtained, the correlation between those Simon effects over the 12 conditions (four blocks and three language conditions) was significant $(r=.76, p<.01, n=12)$.

\section{DISCUSSION}

We observed a reversed Simon effect in the L1 and the combined L1/L2 conditions (see Figure 2). We take this as support for common spatial representations for verbospatial and visuospatial information (Zhang et al., 1999, Figure 1). Before we discuss these data in more detail, we first consider the inducer trials.

Participants responded more slowly to L 2 words than to L1 words, especially in the mixed-language condition. This is consistent with studies on bilingual language processing that show advantages for L1 processing over L2 processing. Van Hell and De Groot (1998), for instance, demonstrated faster lexical decision times for L1 words than for L2 words. It is striking that word RTs are much slower in the combined L1/L2 condition than in any of the separate language conditions. This effect could be caused by the RT cost that is associated with switching between languages (see, e.g., Meuter \& Allport, 1999), but since diagnostic Simon trials also are slower in the combined L1/L2 condition, this effect could also be attributed to general slowing because more stimuli are mapped on the responses in this condition.

The data from the diagnostic trials indicate a significant reversed Simon effect in the RTs of the L1 condition and in the error percentages of the combined L1/L2 condition, whereas Proctor et al. (2000) observed that the Simon effect disappeared while practicing incompatible verbospatial mappings. In general, both studies suggest an influence of verbospatial processes on a visuospatial task and are in line with intermediate shared representations of space. On the other hand, the data also indicate that it is hard to obtain a significant reversed Simon effect, and that it is possible to obtain significant results in RTs for one condition and in error rates for another. The reason for this is most likely the fact that the reversal is a temporary phenomenon and

Table 2

Reaction Times (RTs, in Milliseconds) and Error Percentages (ER\%) for the Diagnostic Trials From the L1, L2, and Combined L1/L2 Conditions, by Blocks

\begin{tabular}{|c|c|c|c|c|c|c|c|c|c|c|c|c|c|c|c|c|c|c|c|c|c|c|c|c|}
\hline \multirow[b]{3}{*}{ Condition } & \multicolumn{8}{|c|}{ L1 } & \multicolumn{8}{|c|}{ L2 } & \multicolumn{8}{|c|}{$\mathrm{L} 1 / \mathrm{L} 2$} \\
\hline & \multicolumn{2}{|c|}{1} & \multicolumn{2}{|c|}{2} & \multicolumn{2}{|c|}{3} & \multicolumn{2}{|c|}{4} & \multicolumn{2}{|c|}{1} & \multicolumn{2}{|c|}{2} & \multicolumn{2}{|c|}{3} & \multicolumn{2}{|c|}{4} & \multicolumn{2}{|c|}{1} & \multicolumn{2}{|c|}{2} & \multicolumn{2}{|c|}{3} & \multicolumn{2}{|c|}{4} \\
\hline & $M$ & $\overline{S E}$ & $M$ & $\overline{S E}$ & $M$ & $\overline{S E}$ & $M$ & $\overline{S E}$ & $M$ & $\overline{S E}$ & $M$ & $\overline{S E}$ & $M$ & $S E$ & $M$ & $S E$ & $M$ & $S E$ & $M$ & $\overline{S E}$ & $M$ & $S E$ & $M$ & $S E$ \\
\hline RT C & 702 & 39 & 640 & 32 & 595 & 28 & 577 & 28 & 652 & 40 & 589 & 33 & 540 & 28 & 528 & 29 & 740 & 39 & 660 & 32 & 595 & 28 & 603 & 28 \\
\hline RT IC & 662 & 33 & 617 & 31 & 579 & 25 & 580 & 25 & 654 & 34 & 618 & 32 & 572 & 26 & 544 & 26 & 712 & 33 & 623 & 31 & 615 & 25 & 610 & 25 \\
\hline RT SIM & \multicolumn{2}{|c|}{-40} & \multicolumn{2}{|c|}{-22} & \multicolumn{2}{|c|}{-15} & \multicolumn{2}{|c|}{3} & \multicolumn{2}{|c|}{2} & \multicolumn{2}{|c|}{29} & \multicolumn{2}{|c|}{32} & \multicolumn{2}{|c|}{16} & \multicolumn{2}{|c|}{-28} & \multicolumn{2}{|c|}{-37} & \multicolumn{2}{|c|}{20} & \multicolumn{2}{|c|}{7} \\
\hline $\mathrm{ER} \% \mathrm{C}$ & 16 & 3 & 10 & 2 & 6 & 1 & 7 & 1 & 8 & 2 & 4 & 1 & 4 & 1 & 3 & 1 & 10 & 2 & 8 & 1 & 8 & 1 & 4 & 1 \\
\hline ER\% IC & 11 & 3 & 7 & 2 & 7 & 1 & 6 & 1 & 8 & 2 & 6 & 2 & 8 & 1 & 8 & 1 & 6 & 2 & 4 & 1 & 4 & 1 & 4 & 1 \\
\hline ER SIM & \multicolumn{2}{|c|}{-5} & \multicolumn{2}{|c|}{-4} & \multicolumn{2}{|c|}{1} & \multicolumn{2}{|c|}{0} & 0 & & 2 & & 2 & & 5 & & - & & - & & - & & - & \\
\hline
\end{tabular}

Note- "RT SIM" refers to the Simon effect in RTs and is the difference between the RTs for incongruent (RT IC) and congruent (RT C) trials. "ER SIM" refers to the Simon effect in error percentages and is the difference between the error percentage for incongruent trials (ER\% IC) and the error percentage for congruent trials $(\mathrm{ER} \% \mathrm{C})$. 


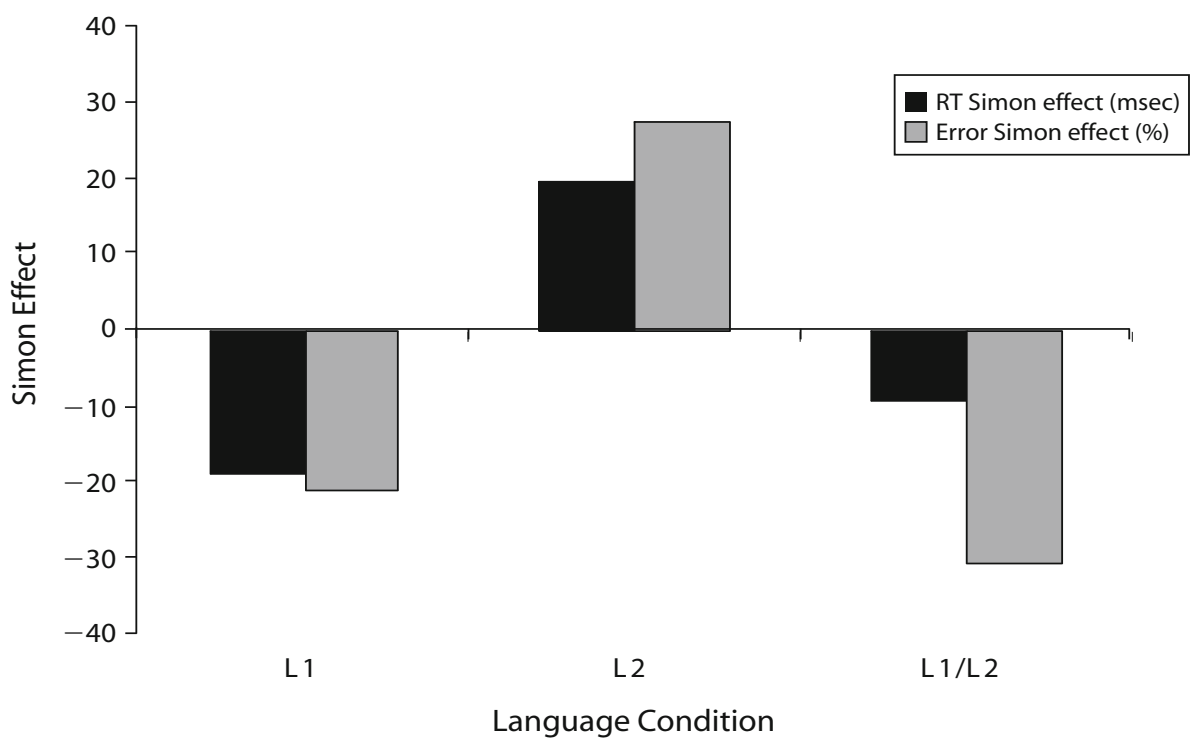

Figure 2. The Simon effect in reaction times (RTs) and error percentages for the three language conditions. The RT Simon effect is the RT for congruent trials subtracted from the RT for incongruent trials. For the Simon effect in error percentages, the percentage error on congruent trials is subtracted from the percentage error on incongruent trials, and for graphical reasons this value is multiplied by 10 .

that the recovery of the regular Simon effect varies over individuals. The correlation between the Simon effects in RTs and error rates, however, indicates no qualitative differences between the measures, and we consequently interpret the reversed Simon effect in the L1 condition (only significant in RTs) and in the combined L1/L2 condition (only significant in error percentages) as similar.

Interestingly, there was no reversal of the Simon effect in L2, so it seems that participants did not strongly activate semantic representations on the basis of L2 words. This is consistent with our assumption of weak lexicalto-conceptual links between L2 words and semantic representations (Figure 1), which follows Kroll and Stewart's (1994) model of bilingual memory.

The observed pattern does not support the idea that combining spatial words from L1 and L2 leads to stronger spatial congruency effects than using only L1 words (De Houwer et al., 2005). In De Houwer et al.'s particular paradigm, this was indeed the case, but our results suggest that their findings do not generalize to the reversal paradigm. Although we cannot directly pinpoint the crucial differences between these paradigms, many discrepancies exist. In the paradigm of De Houwer et al., Dutch native speakers were instructed to say "bee" or "boo" to the words LINKS/GAUCHE ("left" in Dutch and French, respectively) and RECHTS/DROITE ("right" in Dutch and French, respectively). On other trials, participants had to say "bee" or "boo" according to the color of the stimulus appearing on the left or right. Color trial responses were faster when the irrelevant location of the stimulus corresponded to the spatial words that were associated with this response. Thus for instance, "bee" was faster for stimuli on the right when "bee" was the response for the word RECHTS/DROITE ("right"). This extrinsic Simon effect, as they labeled it, was only observed in the condition in which Dutch (L1) and French (L2) were combined. When only L1 words were used, no effect on the color trials was observed. More research is needed to locate the crucial differences between the reversal and extrinsic Simon paradigms.

The Simon effect gradually changed as our experiment proceeded, as indicated by the interaction between block and congruency. Notebaert et al. (2006) also observed that the effect of incompatible magnitude mapping (right response to numbers less than 5 ; left response to numbers greater than 5) was restricted to the first half of their experiment. One possible explanation is that the Simon effect is usually larger for fast RTs, which is explained in terms of decay of the irrelevant spatial information (see, e.g., Hommel, 1994). One could argue that the observed Simon effect is the result of two priming mechanisms: one based on the preexisting compatible associations, and one on the new incompatible mapping rule (Tagliabue, Zorzi, Umiltà, \& Bassignani, 2000). Since we know that the priming effect of the preexisting compatible associations is stronger for fast RTs, it is possible that for later blocks, with faster RTs, this preexisting priming route gains more weight, and the reversal disappears.

An alternative explanation for reversed Simon effects could be formulated on the basis of participants verbalizing the Simon trials in terms of LINKS (left) and RECHTS (right). When participants "name" the location of Simon trials in their first language, it would be unsurprising to observe that these locations become associated with the responses of the verbospatial mapping rule. Note that this explanation was put forward by Proctor et al. (2000) in order to explain the elimination of the Simon effect. Although this explanation could account for the present findings, it would be hard to explain the reversal of the 
SNARC effect, in which it is very unlikely that participants recoded numerical stimuli in terms of left and right (Notebaert et al., 2006). Consequently, although verbalization cannot be excluded in this study, the general picture is more in favor of shared abstract representations.

\section{Conclusion}

In this study, we demonstrated that training a verbospatial incompatible mapping rule reverses the Simon effect. The results provide support for shared spatial representations in which different types of spatial information converge. This representation can be interpreted as a semantic layer intermediate to input and output layers.

\section{AUTHOR NOTE}

Correspondence related to this article may be sent to W. Notebaert, Department of Experimental Psychology, Ghent University, H. Dunantlaan 2,9000 Ghent, Belgium (e-mail: wim.notebaert@ugent.be).

\section{REFERENCES}

Cohen, J. D., Dunbar, K., \& McClelland, J. L. (1990). On the control of automatic processes: A parallel distributed processing account of the Stroop effect. Psychological Review, 97, 332-361.

Dehaene, S., Bossini, S., \& Giraux, P. (1993). The mental representation of parity and number magnitude. Journal of Experimental Psychology: General, 122, 371-396.

De Houwer, J. (2004). Spatial Simon effects with nonspatial responses. Psychonomic Bulletin \& Review, 11, 49-53.

De Houwer, J., Beckers, T., Vandorpe, S., \& Custers, R. (2005). Further evidence for the role of mode-independent short-term associations in spatial Simon effects. Perception \& Psychophysics, 67, 659-666.

DYer, F. N. (1971). Color-naming interference in monolinguals and bilinguals. Journal of Verbal Learning \& Verbal Behavior, 10, 297-302.

FitTs, P. M., \& SEEger, C. M. (1953). S-R compatibility: Spatial characteristics of stimulus and response codes. Journal of Experimental Psychology, 46, 199-210.

Hommel, B. (1994). The relationship between stimulus processing and response selection in the Simon task: Evidence for a temporal overlap hypothesis. Psychological Research, 55, 280-290.
Kornblum, S., Hasbrouce, T., \& Osman, A. (1990). Dimensional overlap: Cognitive basis for stimulus-response compatibility-A model and taxonomy. Psychological Review, 97, 253-270.

KROLL, J. F., \& STEWART, E. (1994). Category interference in translation and picture naming: Evidence for asymmetric connections between bilingual memory representations. Journal of Memory \& Language, 33, 149-174.

LiU, X., Banich, M. T., Jacobson, B. L., \& Tanabe, J. L. (2004). Common and distinct neural substrates of attentional control in an integrated Simon and spatial Stroop task as assessed by event-related fMRI. NeuroImage, 22, 1097-1106.

MACLEOD, C. M. (1991). Half a century of research on the Stroop effect: An integrative review. Psychological Bulletin, 109, 163-203.

Marble, J. G., \& Proctor, R. W. (2000). Mixing location-relevant and location-irrelevant choice-reaction tasks: Influences of location mapping on the Simon effect. Journal of Experimental Psychology: Human Perception \& Performance, 26, 1515-1533.

Notebaert, W., Gevers, W., Verguts, T., \& Fias, W. (2006). Shared spatial representations for numbers and space: The reversal of the SNARC and the Simon effect. Journal of Experimental Psychology: Human Perception \& Performance, 32, 1197-1207.

Proctor, R. W., Marble, J. G., \& VU, K.-P. L. (2000). Mixing incompatibly mapped location-relevant trials with location-irrelevant trials: Effects of stimulus mode on the reverse Simon effect. Psychological Research, 64, 11-24.

Simon, J. R. (1990). The effects of an irrelevant directional cue on human information processing. In S. Proctor \& J. Reeve (Eds.), Stimulus-response compatibility: An integrated perspective (pp. 31-86). Amsterdam: Elsevier.

Tagliabue, M., Zorzi, M., Umiltà, C., \& Bassignani, F. (2000). The role of long-term-memory and short-term-memory links in the Simon effect. Journal of Experimental Psychology: Human Perception \& Performance, 26, 648-670.

Van Hell, J. G., \& De Groot, A. M. B. (1998). Disentangling context availability and concreteness in lexical decision and word translation. Quarterly Journal of Experimental Psychology, 51A, 41-63.

Zhang, H., Zhang, J., \& Kornblum, S. (1999). A parallel distributed processing model of stimulus-stimulus and stimulus-response compatibility. Cognitive Psychology, 38, 386-432.

(Manuscript received January 8, 2007; revision accepted for publication March 27, 2007.) 